\title{
First Steps Towards Defining Galactic Niches
}

\author{
Simon Conway Morris \\ Department of Earth Sciences, University of Cambridge, \\ Downing Street, Cambridge CB2 3EQ, England
}

\begin{abstract}
Terrestrial evolution encompasses many levels, from proteins to societies. Potentially any level can be encompassed by a multidimensional "habitation box", of which only a minute fraction is occupied. On the basis of evidence from evolutionary convergence it is argued that life elsewhere will not only be similarly constrained, but also strangely similar.
\end{abstract}

\section{Introduction}

Enrico Fermi's famous rumination of "Are we alone?" is countered by the unproven and optimistic assumption (based on abundance of interstellar organics, discovery of extra-solar planets, etc., etc.) that life is very widespread. It is assumed, however, that the range of alien diversity matches poorly, if at all, our terrestrial counterpart. A corollary of this is that the myriad of evolutionary trajectories found on other planets will lead to genuinely alien destinations: many have argued that intelligence is as fortuitous and accidental as aardvarks or zygomycetes. About the only thing that universal life might share, and this too is not universally agreed, is that it is "carbaquist", that is carbon and water based.

At first sight this pessimism that Earth's biosphere provides any useful guide to alien diversity and ecology seems entirely justified. If correct, this might give pause for thought as to the validity of SETI. Might it even be that if intelligence evolves elsewhere it will be utterly unintelligible? Such pessimism is based on the simple observation of the combinatorial immensity of biological space, what Walter Elsasser refers to as "immense numbers", typically in excess of $10^{100}$ alternatives (Elsasser 1998). Such a metaphorical hyperspace is defined at any level of interest. This could range from proteins, e.g., imagine all the proteins possible from a string of 100 amino acids each of which may be any one of the twenty amino acids available to life, to societal or cultural complexity (e.g., Cronk 1999). Quite clearly, in the terrestrial context only a minute combinatorial fraction of these hyperspaces can be occupied. Correspondingly this should apply equally to alien biospheres, and so it might also be argued that in each case the zone of "habitation" (at whatever level) will be utterly remote from that found on the Earth. 


\section{Biological Hyperspaces}

That life, at whatever level (proteins to culture), only occupies a tiny part of the total potentially available hyperspace seems to be beyond dispute. My thesis, which if correct has a number of interesting implications, is that practically all of the remaining spaces are permanently uninhabitable: they never have been, and more importantly, never can be nor ever will be occupied. In their interactions with the physico-chemical world these hypothetical alternatives (be they proteins or cultures) are maladaptive. The basis of this argument is evolutionary convergence, which I explore at length elsewhere (Conway Morris 2003). If correct, and it will be obvious I am defining a research programme rather than coming to a definitive conclusion, then it may be that the Earth's biosphere falls within the principle of Copernican mediocrity.

\subsection{Navigation Across Biological Hyperspace}

Before considering the difficulties in defining and connecting the various hyperspace "boxes" that encompass the biosphere, we might note an interesting implication. Even if only a miniscule fraction of a given "hyperspace" is occupied, this does not tell us if there is one principal zone (perhaps with some outliers) or whether there are numerous areas each separated by a "gulf" of unoccupied (and I argue unoccupable) space. In the latter case, given the reality of evolution, then the question arises as to how "navigation" to the remotely situated points of "stability" is ever achieved (Axe 2000). Here too the "worm-holes" through this metaphorical hyperspace may be pre-determined by highly specific circumstances. If this research programme is to be viable it must surmount two obvious problems. The first is the definition of suitable metrics to capture and render tractable a particular "habitation box". The second, and more problematic, is how to interconnect and correlate these boxes: at its most ridiculous if we seek to define a biosphere how do we connect myoglobin to a propensity to monotheism? The simple answer is that for the foreseeable future we cannot. However, the interconnections suggested by genomics and proteomics, algorithms for the search of a given hyperspace, the development of hierarchical methods, and definitions of biological stability should lead us towards the desired paradigm.

The search for niches that might be galactic in their distribution can be considered at several levels, encompassing DNA to culture. Life on Earth, of course, provides the reference point. In at least some cases the ubiquity of evolutionary convergences (Conway Morris 2003) suggests that the solutions to life are indeed limited, sometimes strictly so. As already indicated, there is little information as to the extent to which higher levels of biological organization depend on the lower levels in terms of emergence as against necessary maintenance. The potentially universal nature of biochemistry (Pace 2001) and the general lack of molecular innovation at higher organizational levels suggests, however, that the emergence of complex forms should be unremarkable. In this case, the Rare Earth hypothesis (Ward \& Brownlee 2000), that pond-scum may be widespread and intelligence very rare, seems difficult to sustain.

A more serious problem, again difficult to quantify, concerns what is known as evolutionary incumbency. This is the observation that, once entrenched, an existing system may be very difficult to dislodge. In general, the range 
of habitats, continental separations and oceanic configurations, and fluctuating environments ensure a rich diversity where novelty is an ever-present possibility. The exception to this, however, may be marked by the emergence of complex and aggressive societies, such as eusocial bees and humans, where a significant proportion of resource is more or less permanently appropriated.

\subsection{Galactic Niches}

Restrictions in space allow only a quick overview of what may transpire to be galactic niches: many details are given elsewhere (Conway Morris 2003). The many peculiarities of DNA hint that its role in replication may have few (if any?) counterparts elsewhere. In a remarkable research campaign Albert Eschenmoser and colleagues have been exploring an "etiology of DNA" (Eschenmoser 1999). Most alternative configurations are unconvincing, and even amongst the few "better" variants, they may still fail as viable alternatives. As Eschenmoser remarks the emphasis must be on optimization, not maximization, of function. The question of the occupation of protein "space" is more difficult, not least because as yet very little is known as to how the protein families evolved, so making it speculative as to how easy it is to arrive at a particular "destination". Even so, the evolutionary convergence of such key molecules as rhodopsin and the respiratory proteins (haemoglobin, myoglobin, haemocyanin), the emphasis on transmembrane helical proteins for transduction processes, and the multiple recruitment of crystallins to confer optical transparency suggest that at various levels the possibilities are limited (Conway Morris 2003). The convergence of complex anatomical structures, sensory systems and physiologies are dealt with elsewhere. In brief, the repeated emergence of such features as bipedality, camera-like eyes, and warm-bloodedness may provide at this level useful guides to more general biological properties. So too the emergence of complex behaviours and organizations show recurrent patterns that encourage the idea that there are indeed wider commonalities. For example, convergence in such features as agriculture, eusociality and societal organization, e.g., fission-fusion (dolphin, chimp), matriarchal (elephant, sperm whale), is again consistent with a restricted range of biological possibilities. So too with intelligences; Lori Marino, for example, has discussed striking convergences between dolphin and primate intelligences (Marino 2002). The independent evolution of such features as cultural transmission and vocalization are also directly relevant to the conclusion that the Earth's biosphere is not unrepresentative.

\section{Conclusion}

The implications of this work are interesting. At the least it suggests a road towards a more general biological theory that might help to reconcile evolutionary theory (the flux of change) with the striking stability imposed by adaptation, the latter being the effectively platonic view of archetypes. At the most it might indicate that if SETI is ever successful, which for other reasons I doubt, then the alien biosphere will spring few surprises, at least to the biologist. Whether the familiarity of such a biosphere will provide much comfort is a topic beyond this paper. 
Acknowledgments. I thank the organizers of BioAstronomy 2002, especially Ray Norris and Carol Oliver, the Royal Society, and St John's College, Cambridge, for support. I also acknowledge the work of many colleagues and thank Sandra Last for technical assistance.

\section{References}

Axe, D. D. 2000, J. Mol. Biol., 301, 585

Conway Morris, S. 2003, Life's Solution: Inevitable Humans in a Lonely Universe, (Cambridge: Cambridge Univ. Press)

Cronk, L. 1999, That Complex Whole: Culture and the Evolution of Human Behavior, (Boulder: Westview Press)

Elsasser, W. M. 1998, Reflections on a Theory of Organisms: Holism in Biology, (Baltimore: Johns Hopkins Univ. Press)

Eschenmoser, A. 1999, Science, 284, 2118

Marino, L. 2002, Brain Behav. Evol., 59, 21

Pace, N. R. 2001, Proc. Natl. Acad. Sci. USA, 98, 805

Ward, P. D., \& Brownlee, D. 2000, Rare Earth: Why Complex Life is Uncommon in the Universe, (New York: Copernicus) 